\title{
Editorial
}

\section{Complex Discrete Nonlinear Dynamics: Chaos and Its Applications}

\author{
Qingdu Li, ${ }^{1}$ Timothy J. Healey, ${ }^{2}$ Xiao-Song Yang, ${ }^{3}$ and Xiaofeng Liao ${ }^{4}$ \\ ${ }^{1}$ Key Laboratory of Network Control \& Intelligent Instrument, Ministry of Education, Chongqing University of Posts and \\ Telecommunications, Chongqing 400065, China \\ ${ }^{2}$ Department of Mathematics, Cornell University, Ithaca, NY 14850, USA \\ ${ }^{3}$ Department of Mathematics, Huazhong University of Science and Technology, Wuhan 430074, China \\ ${ }^{4}$ School of Electronic and Information Engineering, Southwest University, Chongqing 400715, China
}

Correspondence should be addressed to Qingdu Li; liqd@cqupt.edu.cn

Received 31 July 2013; Accepted 31 July 2013

Copyright (C) 2013 Qingdu Li et al. This is an open access article distributed under the Creative Commons Attribution License, which permits unrestricted use, distribution, and reproduction in any medium, provided the original work is properly cited.

Nonlinear and chaotic behavior appears widely and naturally in many discrete dynamical systems; its study has led to vast multidisciplinary research in fields ranging from the natural sciences (mechanics, chemistry, biology, ecology, etc.) to the social sciences (economics, sociology, etc.) and engineering (electronics, control, communication, security, etc.). Recent advances in dynamical systems theory and computational methodologies have provided new ways of understanding such complex phenomena via novel tools and applications of discrete nonlinear dynamics.

This special issue contains ten papers, of which eight study chaotic behavior in some practical models from interesting fields, such as a Cournot-Bertrand duopoly game model, a price game model of irrigation water, a susceptibleinfected-susceptible (SIS) model, the credit risk transfer (CRT) market model, a tumor growth model, a fractionalorder system, a small neural network, and rectangular plates. There are also two theoretical papers focusing on the bifurcation of limit cycles from periodic orbits and the existence and uniqueness of the global solution for the stochastic Boussinesq equations, respectively.

Two game models are studied in detail in this special issue as typical discrete-time systems. "Complexity analysis of a Cournot-Bertrand duopoly game model with limited information" by $\mathrm{H}$. Wang and J. Ma proposes a Cournot-Bertrand mixed game model, where the market has linear demand and two firms have the same fixed marginal cost, and shows complex dynamics, such as bifurcation scenarios and route to chaos, as well as parameter basin by numerical experiment. "Bifurcation and chaos in a price game of irrigation water in a coastal irrigation district" by B. Xin and Y. Li emphatically discusses the stability and codimension-two period-doubling (flip) bifurcation in a price game model with numerical bifurcation-based continuation methods, and the 0-1 test algorithm is used to compute the median value of correlation coefficient to indicate chaotic dynamics.

Mathematical models in the real world are often timecontinuous systems or hybrid systems. To study their nonlinear dynamics, one frequently converts them to discrete systems. A common method is discretizing time with a well-chosen stepsize. "Bifurcation and chaotic behavior of a discrete-time SIS model" by J. Li and N. Cui obtains a discretetime epidemic model with the Euler method, and shows rich and complex dynamical behaviors, such as transcritical bifurcation, flip bifurcation, Hopf bifurcation, and chaos. "Dynamics evolution of credit risk contagion in the CRT market" by T. Chen et al. introduces a nonlinear dynamics model to depict the dynamic behavior characteristics of evolution of credit risk contagion through numerical simulation and reports a series of Hopf bifurcations and chaotic phenomena in the process of credit risk contagion. "Modeling a tumor growth with piecewise constant arguments" by F. Bozkurt constructs two models of a tumor growth and shows that the increase of the population growth rate 
decreases the local stability of the positive equilibrium point. "A fractional-order chaotic system with an infinite number of equilibrium points" by P. Zhou et al. studies chaos in a new 4-dimensional fractional-order system, which has an infinite number of equilibrium points, and also presents a chaotic synchronization scheme.

Another common method to discretize time-continuous systems is using Poincaré return maps. "Multipulse heteroclinic orbits and chaotic dynamics of the laminated composite piezoelectric rectangular plate" by $\mathrm{M}$. Yao and W. Zhang demonstrates how to employ the energy phase method to analyze the Shilnikov type multipulse heteroclinic bifurcations and chaotic dynamics of high-dimensional nonlinear systems in engineering applications. "Horseshoe chaos in a $3 d$ neural network with different activation functions" by F. Yang et al. studies a small neural network with three neurons. Although the network is a simple hybrid system with all subsystems being exponentially stable, they find that the network can exhibit very complex dynamics such as limit cycles and chaos, which implies that chaos is more related to its weight matrix than the type of activation functions.

Limit cycles, as well as their number and distribution, are significant for understanding the geometrical structures of the state space. "Bifurcation of limit cycles of a class of piecewise linear differential systems in $R^{4}$ with three zones" by Y. Cheng studies the bifurcation of limit cycles from periodic orbits of a four-dimensional system by the averaging method, and shows that when the parameter is sufficiently small, at most six limit cycles can bifurcate from periodic orbits in a class of asymmetric piecewise linear perturbed systems.

The existence and uniqueness of the solution are a fundamental problem for each dynamical system. "Perturbation of stochastic Boussinesq equations with multiplicative white noise" by C. Yang and X. Zhao studies the Boussinesq equations perturbed by multiplicative white noise, and shows the existence and uniqueness of the global solution. They also obtain some regularity results for the unique solution.

The topics of the selected papers in this issue do not of course cover this entire multidisciplinary field. Rather they represent rich and many-faceted results that we have the pleasure of sharing with the readers.

Since most dynamical systems have no explicit solution, it is often a hard task to theoretically analyze complex discrete nonlinear behavior. Although numerical methods works for most systems, it is often criticized because of numerical errors and increasing computational cost. Combining theoretical and numerical studies, like some of the aforementioned papers is perhaps a powerful approach. With interval algorithms on massive computing devices, nonlinear behavior, such as limit cycles, homoclinic/heteroclinic orbits, and even chaos, can be studied rigorously, and the complex geometrical structure of the state space and the parameter space may also be analyzed to reveal mechanisms behind the complex behavior, which are important for scientific and engineering purposes.

\section{Acknowledgments}

We would like to express our appreciation to the authors for their excellent contributions and patience in assisting us. The fundamental work of all reviewers on these papers is also very greatly acknowledged. In addition, $\mathrm{Q}$. Li also would like to thank the National Natural Science Foundation of China (61104150) and Science Fund for Distinguished Young Scholars of Chongqing (cstc2013jcyjjq40001).

Qingdu Li Timothy J. Healey Xiao-Song Yang Xiaofeng Liao 


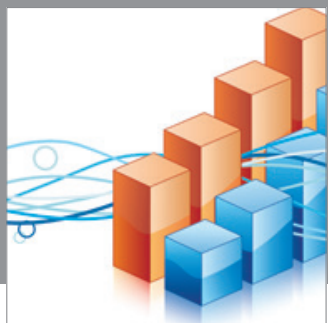

Advances in

Operations Research

mansans

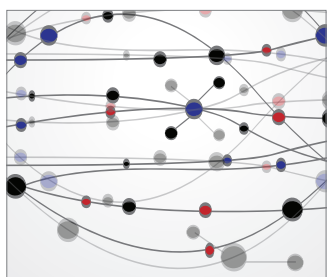

The Scientific World Journal
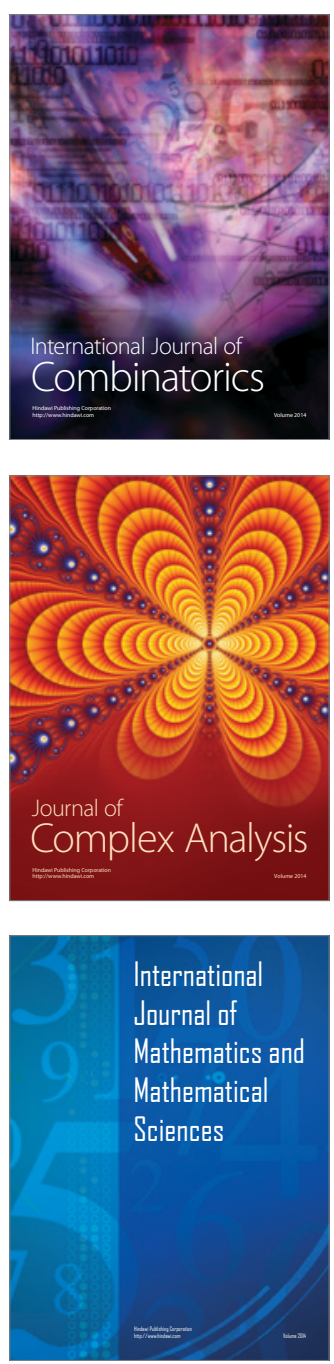
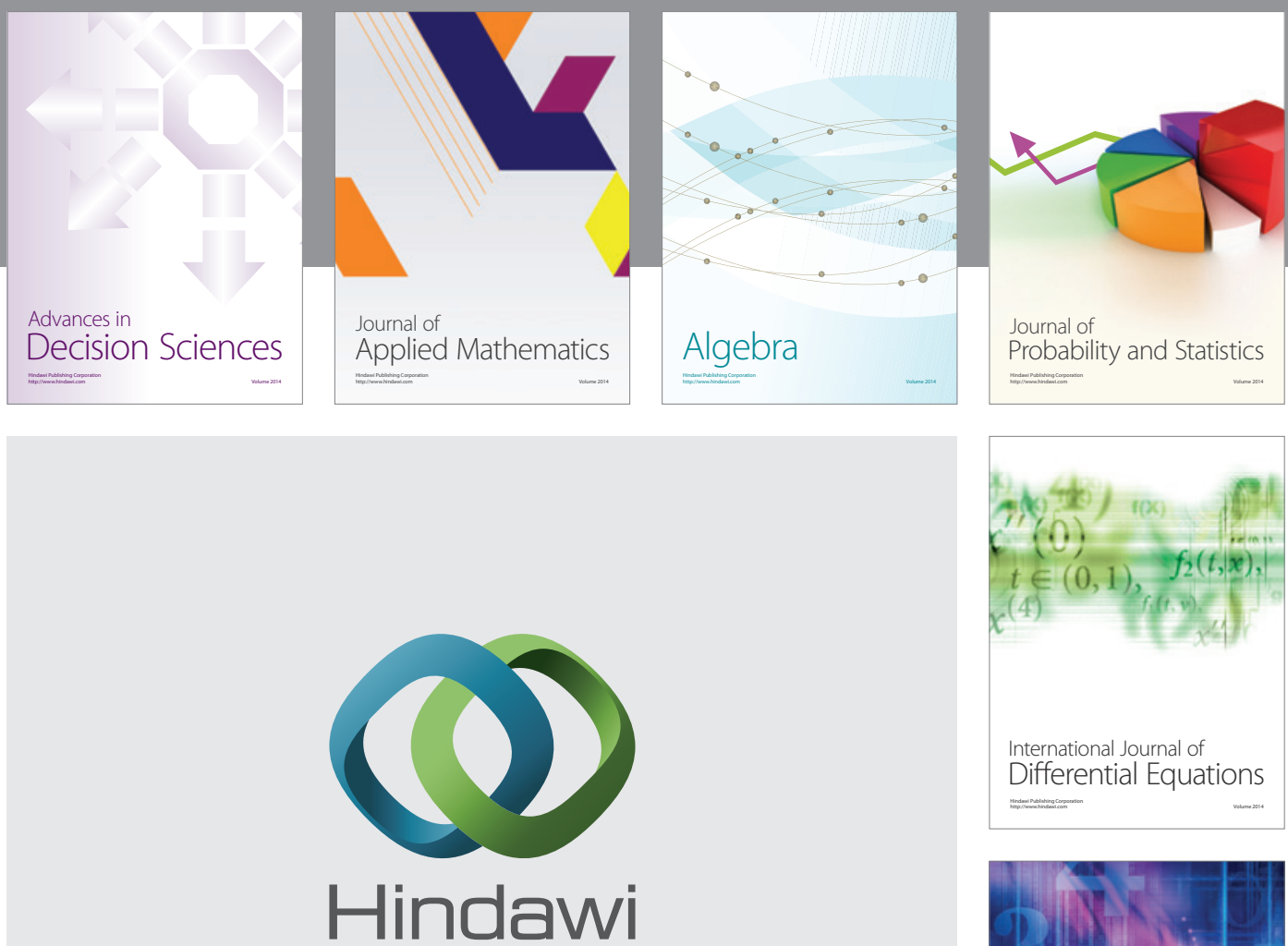

Submit your manuscripts at http://www.hindawi.com
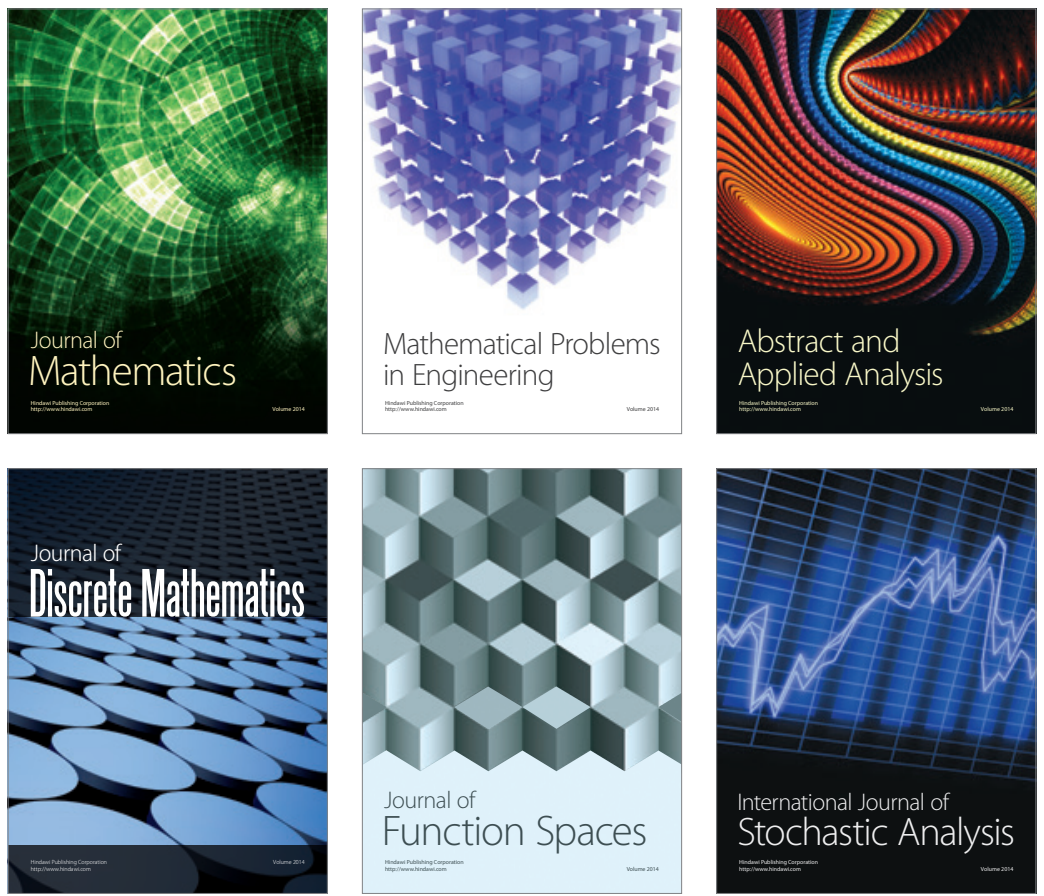

Journal of

Function Spaces

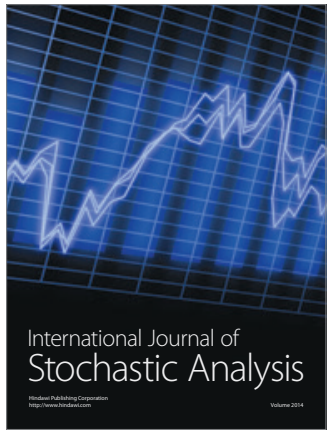

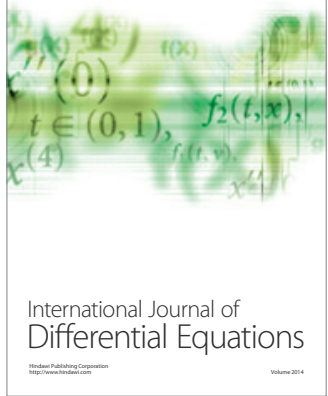
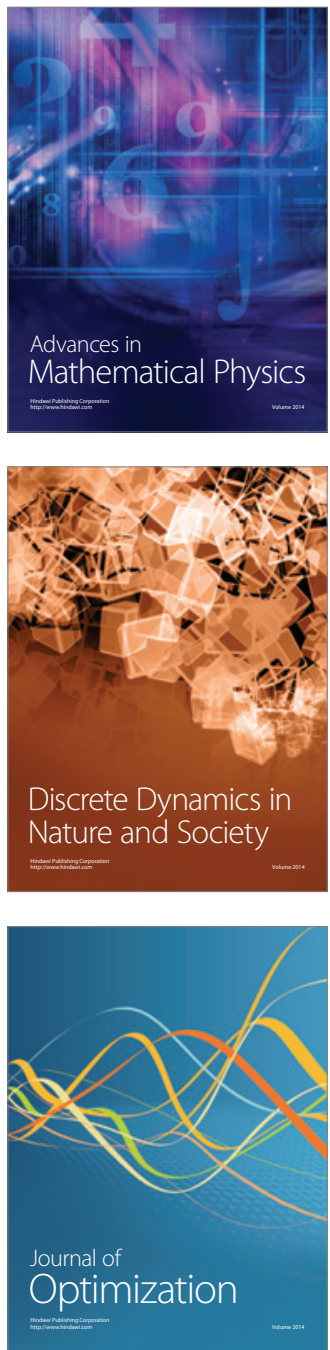